\title{
A high-efficiency and ultrathin transmission-type circular polarization converter based on surface structure
}

\author{
Peng $\mathrm{Xu}^{1}$, Wei Xiang Jiang ${ }^{1, *}$, Xiao $\mathrm{Cai}^{2}$, Yue Gou ${ }^{1}$, and Tie Jun Cui ${ }^{1}$ \\ ${ }^{1}$ State Key Laboratory of Millimeter Waves, School of Information Science and Engineering, Southeast University, \\ Nanjing 210096, PR China \\ ${ }^{2}$ Research Center of Applied Electromagnetics, Nanjing University of Information Science and Technology, Nanjing 210044, \\ PR China
}

Received: 9 November 2020 / Accepted: 2 January 2021

\begin{abstract}
In this paper, we propose, design and fabricate a kind of ultrathin and high-efficiency circularly polarization converter based on artificially engineered surfaces in the transmission mode. The converter is composed of double-layer periodic surface structures with cross slots. The top and bottom layers are printed on both sides of the F4B substrate and connected by metallic via holes. The proposed converter can transform the right-handed circularly polarized incident electromagnetic (EM) wave to a left-handed circularly-polarized one with near-unity efficiency in the transmission mode, or vice versa. We explain the conversion mechanism based on numerical simulations and equivalent circuit (EC) theory. The measured result has a good agreement with the simulated one in the working frequency band. Such ultrathin polarization converters can be used in wireless microwave communication, remote sensing, and EM imaging where circularly polarization diversity is needed.
\end{abstract}

Keywords: Circular polarization converter / high-efficiency and ultrathin / transmission type / equivalent circuit

\section{Introduction}

Circularly polarization $(\mathrm{CP})$ is one of the most important characteristics of EM waves and CP has always attracted the attention of researchers in wireless and satellite communication [1]. The manipulation of $\mathrm{CP}$ converter is the ability to convert the polarization state of incident waves when it reflects or transmits through polarization converters, including linear-to-circular (LTC) polarization converter, which requires the generation of two orthogonal field components with a $90^{\circ}$ phase shift between them, and circular-to-circular (CTC) polarization converter $[2,3]$. The realization of $\mathrm{CP}$ waves has been a challenging and hard task. Traditional ways to achieve $\mathrm{CP}$ waves can be directly created by antenna [4]. Another alternative approach adopted in recent years is to use an LTC polarization converter positioned in front of the antenna, which radiates linearly polarized EM waves $[5,6]$. CP converter can convert incident waves with given right- or left-hand circularly polarized (RHCP or LHCP) state to versa state ones in the reflected or transmitted mode.

\footnotetext{
* e-mail: wxjiang81@seu.edu.cn
}

Generally, conventional polarization devices are usually applied to generate the desired polarized state, and can also be used to minimize the Faraday effects [7]. It was popular to control the polarization of the EM wave using bulky wave-plate or anisotropic materials [8].

Recently, artificially engineered surfaces have been presented to design polarization converter which is capable of transforming the polarization of an incident wave to other polarization states [9,10]. Due to exotic EM properties, artificial metasurface has the advantages of low profile and ease of fabrication so that it has a wide range of applications in many areas of science and engineering. The metasurface-inspired structures have been used to design linear polarization converter [11], circular polarization converter [12], linear and circular polarization converter [13], and linear and vortex waves converter [14]. Most designs of the polarization converters are multilayer planar structures or 3-D structures, which are based on the slot resonators [15], meander lines [16,17], and patch or wire gird configurations [18-20]. The linear-toelliptical polarization converters have also been presented and verified in the microwave and Mid-infrared bands [21]. However, bulky and complex are costly and inconvenient. Hence, it is still a challenge to develop polarizationconversion devices with a small thickness and lightweight. 
(a)

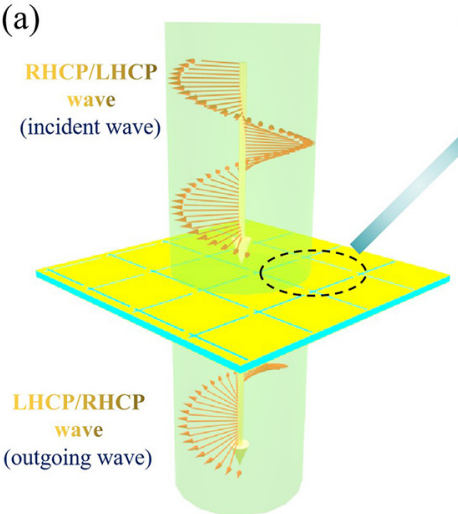

(b)

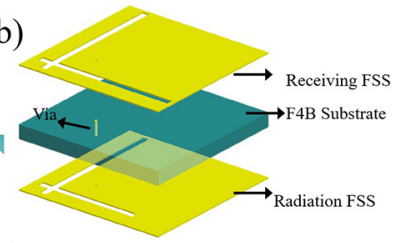

(c)

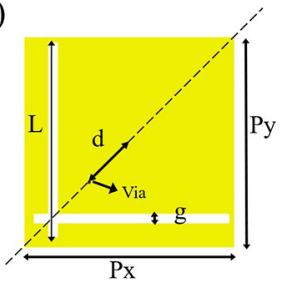

Fig. 1. Illustration of polarization converters. (a) Intuitive scheme of the polarization converter array; (b) Element of the polarizer; (c) Top view of the unit cell.

In this paper, a novel ultrathin and high-efficiency circularly polarization converter is proposed and demonstrated in the transmission mode. The converter can realize transmission polarization conversion from right-handed circularly polarized incident EM wave to left-handed circularly polarized one. The polarizer is constructed of a periodic array of the dielectric substrate sandwiched with artificial surface structures. The top and bottom layers are composed of cross-slot structures printed on both sides of the ultrathin F4B substrate and connected by metallic vias. The conversion mechanism is explained by full-wave numerical simulations and equivalent circuit theory. The measured results and simulated ones have good agreement in the entire frequency range.

\section{Design}

Figure 1 shows the sketch of the proposed transmitted polarization converter, which can realize transmission polarization conversion from right- or left-handed circularly polarized incident EM wave to left- or right-hand circularly polarized one. Note that we consider and discuss the case when the right-handed circular-polarization wave incident is a special example here and hereinafter. The polarizer is an array of artificial structures. The built unit cell is composed of the F4B dielectric substrate, receiving surface, and radiation surface, as shown in Figure 1b.

Here, the receiving surface and radiation surface are printed on both sides of the entire $\mathrm{F} 4 \mathrm{~B}$ substrate and connected by one metallic via. When a circularly polarized wave is incident, the receiving surface can effectively capture the incident wave. Furthermore, the captured energy can be transmitted to the radiation surface through the metallic via. Also, the receiving surface and radiation surface are both composed of metallic patches and a crossslot on the surface, as shown in Figure 1c. The F4B substrate with permittivity $\varepsilon_{\mathrm{r}}=2.65$, loss tangent tan $\delta=0.001$, and thickness $h=2 \mathrm{~mm}$ is sandwiched with artificial structures. The simulations and optimization calculations are carried out using commercial software, Computer Simulation Technology (CST), and the frequency domain

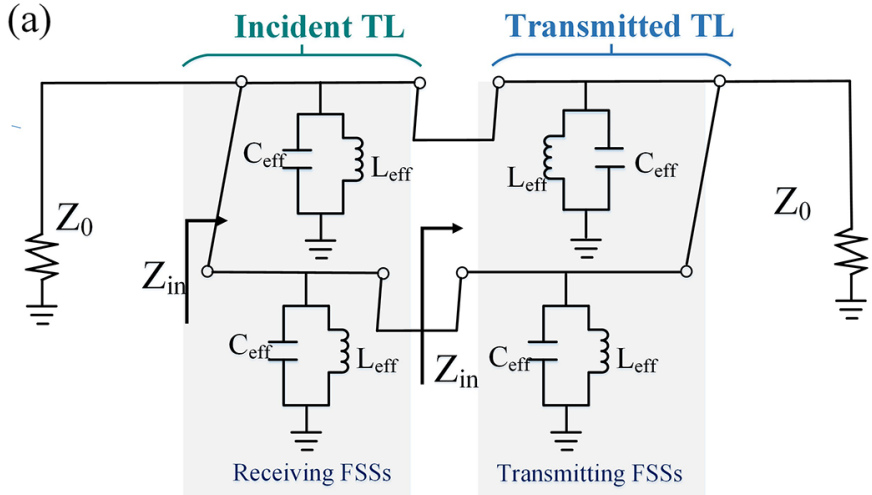

(b)

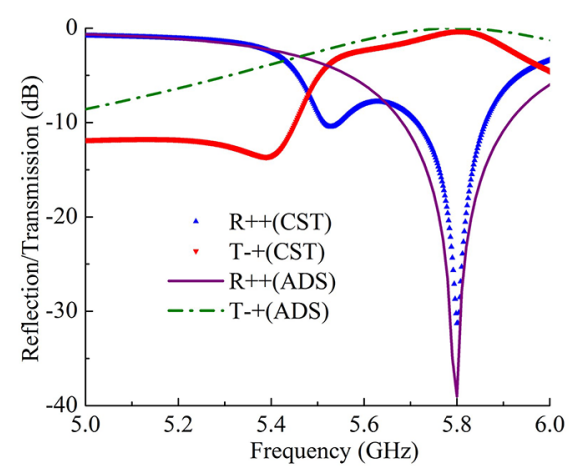

Fig. 2. (a) Equivalent circuit model of the unit cell; (b) S-parameter of the element of obtained from the equivalent circuit model and numerical simulation.

solver was chosen with periodic boundary conditions in $\mathrm{x}$-direction and $\mathrm{y}$-direction and the Floquet port in the $\mathrm{z}$-direction to extract scattering parameters. The dimensions of the unit cell in Figure 1c are $P_{x}=22 \mathrm{~mm}, P y=22 \mathrm{~mm}, L=$ $20.5 \mathrm{~mm}, g=1 \mathrm{~mm}, d=5.94 \mathrm{~mm}$, and the diameter of via hole is $0.4 \mathrm{~mm}$. The proposed polarization converter is designed to operate at $5.8 \mathrm{GHz}$ in the transmission mode. The artificial structures are made of the copper film with a thickness of $t=0.035 \mathrm{~mm}$, and hence the total thickness of the polarizer is $2.07 \mathrm{~mm}$, about $1 / 25$ wavelengths.

Each element based on the incident and transmission wave modes can be equivalent to an EC, as illustrated in Figure 2a. Essentially, the incident wave and the radiated wave can be both treated as the transmission-line (TL) model in the propagation direction and the EC model is a parallel LC circuit. Here, the inductance is associated with the electric current flowing in the surface structure and the capacitance is generated by the cross-slot. The incident RHCP wave can be well converted into the LHCP one in the transmission mode, at the resonant frequency $5.8 \mathrm{GHz}$, by the metallic via due to the symmetry between the receiving and transmitting transmission line. The values of capacitance and inductance can be calculated by the stored electromagnetic energies within the conversion structure $[22]$.

According to TL theory, when the input impedance $\left(Z_{i n}\right)$ matches the out impedance $\left(Z_{0}=377 \Omega\right)$ [23], we have

$$
Z_{\text {in }}=\frac{Z_{0}}{2 Z_{0}\left(Z_{L}+Z_{C}\right)+1}=Z_{0}
$$


(a)



(b)

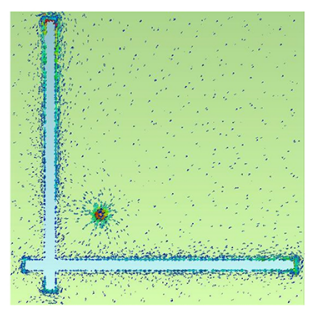

(c)

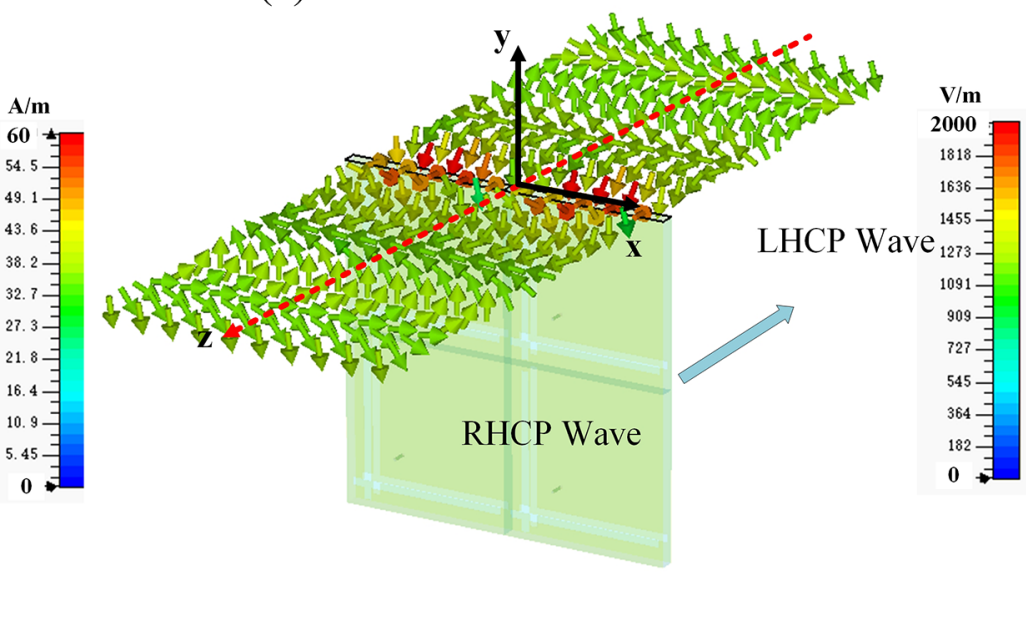

Fig. 3. Simulated surface current distributions at (a) $5.8 \mathrm{GHz}$; (b) $5 \mathrm{GHz}$; (c) Electrical vector variation.

in which,

$$
Z_{L}=j \omega L_{e f f}, Z_{C}=-j \frac{1}{\omega C_{e f f}}
$$

In such a case, the resonant frequency is

$$
\omega_{0}=\frac{1}{\sqrt{L_{e f f} C_{e f f}}} \rightarrow f_{0}=\frac{1}{2 \pi \sqrt{L_{e f f} C_{e f f}}} .
$$

The values of the inductance and capacitance of the polarizer element in infinite array form can be expressed by [24]

$$
C_{e f f}=\frac{\left|I_{C}\right|^{2}}{4 \omega^{2} \bar{W}_{e}}, L_{e f f}=\frac{4 \bar{W}_{m}}{\left|I_{L}\right|^{2}}
$$

where $I_{L}$ and $I_{C}$ stand for the currents of the inductance and capacitance, respectively. In particular, it is noted that we ignore the loss of the F4B substrate and consider the metallic plate as a perfect electric conductor in the calculation. For the unit cell of the proposed CP converter, the stored electrical energy can be calculated by the Floquet mode of the frequency domain solver in CST. We get the approximate values of $L_{\text {eff }}=2.464 \mathrm{nH}$ and $C_{\text {eff }}=0.306 \mathrm{pF}$. Figure $2 \mathrm{~b}$ shows the reflection and transmission coefficient of the circuit model in the Advanced Design System (ADS) is compared to the simulation result in CST. From the result shown in Figure 2b, it is observed that the EC model of the presented polarizer is validated, and the relative errors are acceptable. In the ADS simulation, the equivalent circuit operates in the lossless state, and the S-parameter curve is smooth. However, in the full-wave simulation, the model works in the lossy state and the coupling between the receiving and radiation FSSs exists, hence, there are some disfferences between the simulated results based on the equivalent circuit model and the full-wave simulation results.
To understand the working mechanism of the presented cross-slot polarization converter, we calculate the induced surface current distributions which are depicted at two typical frequencies, as shown in Figures $3 \mathrm{a}$ and $3 \mathrm{~b}$. The front cross-slot surface structure responses to the incident circular-polarization wave with one handedness and the strong current intensity have been induced at resonant frequency $5.8 \mathrm{GHz}$, as illustrated in Figure $3 \mathrm{a}$. The propagating current is converted into a radiated circularly polarization wave with another handedness by the backside cross-slot surface structure. For clarity, when a righthanded circular-polarization wave is normally incident onto the sample, a strong current, moving around the top layer structure, will flow to the bottom layer through the via, and the bottom layer will radiate the left-handed circular-polarization wave due to the discontinuity of current. We remark that the current distribution on the bottom layer is consistent with that on the top layer due to the symmetry of the structure. A dramatic attenuation of the current intensity has been observed when the frequency has a little shift, as shown in Figure 3b. To better reveal how the incident right-handed circular-polarization wave on the input port surface is fully converted into the transmitted left-handed circular-polarization wave on the output port, the electrical vector variation is shown in Figure 3c. It is observed that the transmission wave has almost the same magnitude as the incident waves, hence the presented converter can achieve an almost perfect polarization conversion.

\section{Result and discussions}

Here, we define the reflection and transmission coefficients as $R_{\mathrm{mn}}$ and $T_{\mathrm{mn}}$, respectively, where the $m$ and $n$ correspond to the polarization states of the transmitted and incident EM waves. For the circular polarization wave, we assume the handedness of the right-handed polarization wave is + (represented by $m$ or $n$ ), and the handedness of 

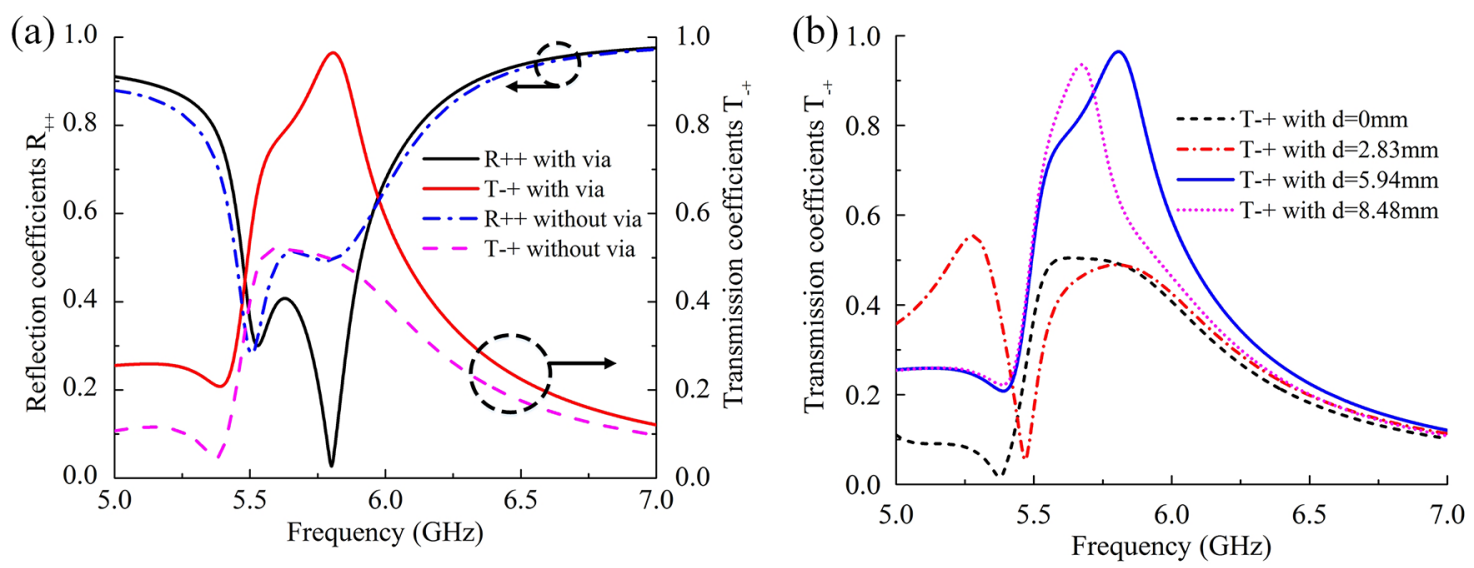

Fig. 4. Simulation results. (a) Transmission performances of the single-layer polarizer with and without via; (b) Transmission performances of single-layer polarizer when the thickness of substrate changes.

(a)



(b)

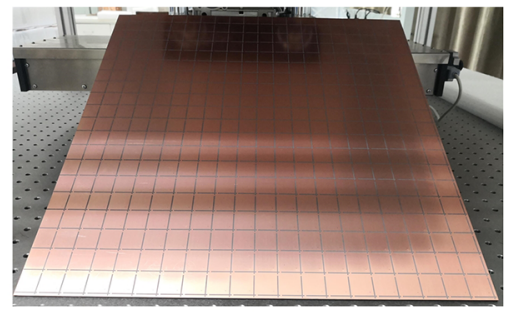

(c)

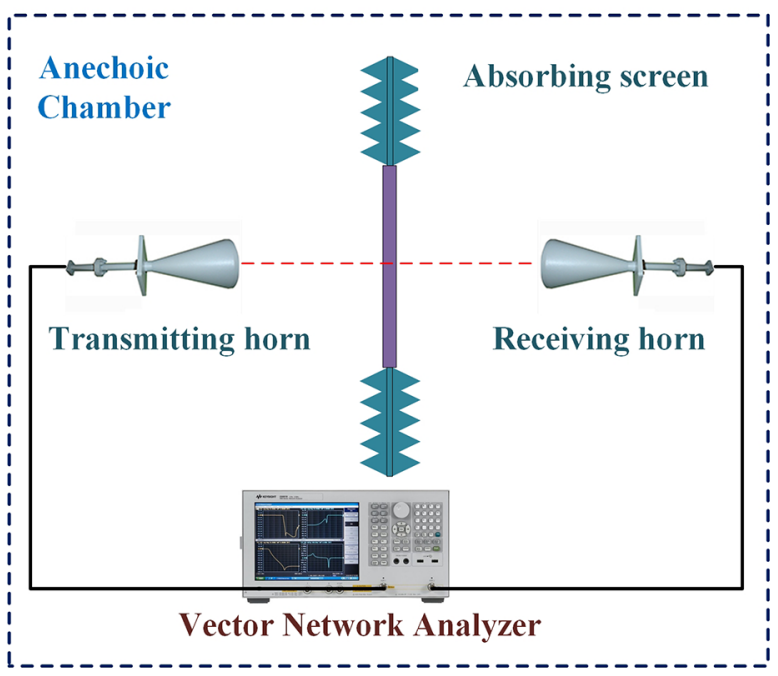

Fig. 5. Photograph of the fabricated sample (a) Top view; (b) Bottom view; (c) Experiment setup. Transmitting horns and receiving horns are used to emit incident waves and receive transmission waves.

the left-handed polarization wave is - (represented by $m$ or $n$ ), respectively. To verify the performance of the proposed design, we calculate the scattering coefficients numerically. The performances of the unit cell of the ultrathin polarizer with and without via were indicated by the results of the simulated co-polarization reflection coefficient $R_{++}$and the cross-polarization transmission coefficient $T_{-+}$, as shown in Figure 4a. It is observed that conversion efficiency $T_{-+}$with via is 0.965 (almost equals to 1 ) and $R_{++}$with via is close to 0 for the designed polarizer at the resonant frequency $5.8 \mathrm{GHz}$. Especially, we ignored the $R_{--}$and $T_{+-}$due to the symmetry of the ultrathin structure for the normal incident. The simulation results of the same element configuration without via are also illustrated in Figure 4a, which reflects the incident waves. In our design, the ultrathin thickness of F4B is $2 \mathrm{~mm}$. Furthermore, we analyze the effect of the position of via (distance from the center of the structure on the diagonal, $d$ ) on the performance of the polarization converter. The transmission coefficients were calculated with different values of $d$, as shown in Figure 4b. It is observed that when $d$ is less than $5.94 \mathrm{~mm}$, the transmission coefficient dramatically, and when $d=5.94 \mathrm{~mm}$, the transmission coefficient reaches to peak, and when $d$ is greater than 5.94, the transmission coefficient decrease gradually. Note that if the radius of the via hole changes, the characteristic impedance of the structure will change, leading to the fluctuation of the S-parameter. When the length of the cross-slot changes, the working frequency will shift. However, the tiny change of the width of the slots has little influence on S-parameter.

To experimentally verify the designed polarizer, we fabricated a sample of the converter by utilizing the conventional printed circuit board (PCB) with the same structural parameters as the simulation model, as shown in Figures 5a and 5b. As mentioned earlier, the operation frequency is designed at $5.8 \mathrm{GHz}$. The sample has dimensions of $440 \mathrm{~mm} \times 440 \mathrm{~mm}$, containing $20 \times 20$ unit cells, and is verified by both the measured complex S-parameters in a microwave anechoic chamber and the 

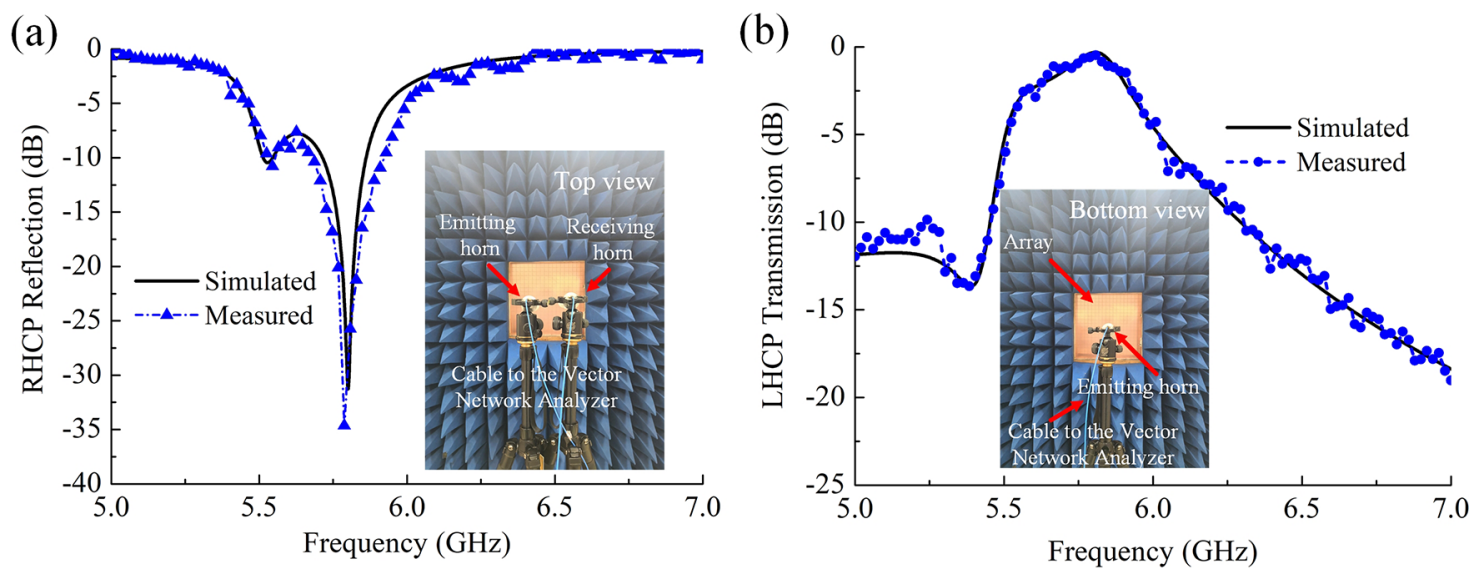

Fig. 6. (a) Co-polarization reflection and (b) cross-polarization transmission obtained by the full-wave simulation and experimental measurement.

full-wave simulations, as illustrated. Also, Figure 5c shows the measurement setup, in which two standard gain spiral CP horn antennas are connected to a vector network analyzer. Here, the transmitting horn is used to generate the RHCP wave at the normal incidence, and the receiving horn serves as a receiver for the co-polarization reflection wave and the cross-polarization transmission wave, respectively. It is noted that the fabricated sample is surrounded by absorbing materials to avoid the diffracted wave. The center height of the polarizer should be the same as the center of the transmitting and receiving antennas so that the high efficiency of receiving and transmitting EM waves are generated. To generate quasi-plane $\mathrm{CP}$ wave excitation of $\mathrm{CP}$ converter, the transmitting horn and receiving horn both were positioned at a distance $d_{c}=100 \mathrm{~cm}$ away from the sample.

The magnitudes of the reflection co-polarization coefficients and transmission cross-polarization coefficients, respectively, were measured at normal incidents, which compared with a simulated one, as shown in Figures $6 \mathrm{a}$ and $6 \mathrm{~b}$. Here, the measured coefficients are normalized to the area of air and PEC with the same dimensions as the sample. It is observed that the measured results agree very well with the simulation results, and the small deviations are likely caused by tolerance in fabrication and measurement. From Figure 6a, we can find that the RHCP reflection coefficient reaches $-31.7 \mathrm{~dB}$ for measurement at $5.79 \mathrm{GHz}$ and $-34.6 \mathrm{~dB}$ for simulation at $5.8 \mathrm{GHz}$, respectively. Also, the LHCP transmission coefficients curve reaches a peak, $-0.3 \mathrm{~dB}$ for simulation at $5.8 \mathrm{GHz}$ and $-0.41 \mathrm{~dB}$ for measurement at $5.79 \mathrm{GHz}$, respectively, as shown in Figure 6b. Due to the peripheral unit cell, the marginal effect of the finite array cannot be avoided in the process of the measurement. The observing frequency ranges from 5 to $7 \mathrm{GHz}$, and the simulation and measured results have a good agreement in the entire frequency range.

To indicate the robustness of the proposed converter, we change the incident angle $(\theta)$, as shown in Figure 7, of the exciting plane wave. It is observed that the conversion efficiency starts to decrease and the resonant frequency

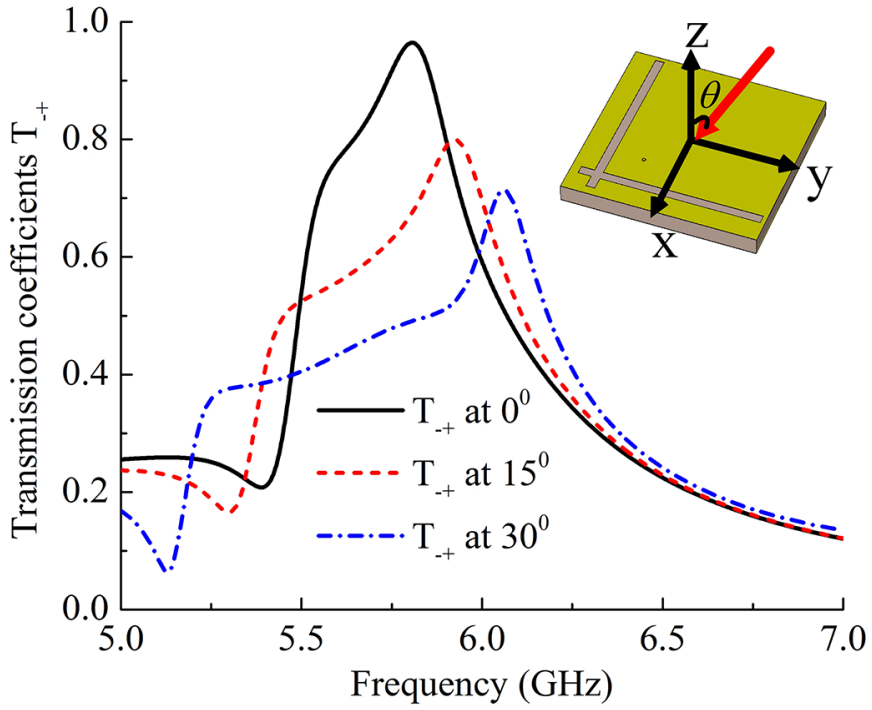

Fig. 7. Transmission coefficients for the simulated at different angles. When the incident angle is normal $\left(0^{\circ}\right)$, the resonant frequency is $5.8 \mathrm{GHz}$ and conversion efficiency is $96.5 \%$; when the incident angle is $15^{\circ}$, the resonant frequency shifts to $5.91 \mathrm{GHz}$ and conversion efficiency is $82.3 \%$, and when the incident angle is $30^{\circ}$, the resonant frequency shifts to $6.06 \mathrm{GHz}$ and conversion efficiency is $71.2 \%$.

shift towards high frequency with the incident angle increases. In Figure 7, we observe that when the incident angle is normal $\left(0^{\circ}\right)$, the resonant frequency is $5.8 \mathrm{GHz}$ and conversion efficiency is $96.5 \%$; when the incident angle is $15^{\circ}$, the resonant frequency shifts to $5.91 \mathrm{GHz}$, and conversion efficiency is $82.3 \%$, and when the incident angle is $30^{\circ}$, the resonant frequency shifts to $6.06 \mathrm{GHz}$ and conversion efficiency is $71.2 \%$.

\section{Conclusion}

In summary, we proposed, fabricated, and investigated a kind of ultrathin circular polarization converter in the transmission mode. The polarizer with a thickness 
of $1 / 25$ wavelengths, at the desired resonant frequency, can achieve extremely high conversion efficiency for incident CP waves with either right or left-handedness. The simulated and measured results agree very well. In the further design, we will consider the phase gradient which can be used to integrate polarization manipulation and phasefront tailoring [25-28].

This work was supported by the National Key Research and Development Program of China under Grant Nos. 2017YFA0700201, 2017YFA0700202, 2017YFA0700203, the National Science Foundation of China under Grant Nos. 61522106, 61631007, 61571117, 61501112, 61501117, 61722106, 61701107, and 61701108, and 111 Project under Grant No.111-2-05, the Scientific Research Foundation of Graduate School of Southeast University under Grant No.YBPY1956.

\section{References}

1. A. Kajiwara, IEEE Trans. Veh. Technol. 44, 487 (1995)

2. S.Y. Wang, W. Liu, W. Geyi, Appl. Phys. B 124, 126 (2018)

3. N. Shao, S.Y. Wang, W. Geyi, Appl. Phys. A 124, 525 (2018)

4. X. Zhang, L. Zhu, IEEE Trans. Antennas Propag. 64, $2172(2016)$

5. E. Arnaud, R. Chantalat, M. Koubeissi, T. Monediere, E. Rodes, M. Thevenot, IEEE Antennas Wireless. Propag. Lett. 9, 215 (2010)

6. X.L. Ma, W.B. Pan, C. Huang, M.B. Pu, Y.Q. Wang, B. Zhao, J.H. Cui, C.T. Wang, X.G. Luo, Adv. Opt. Mater. 2, 945 (2015)

7. T. Meissner, F.J. Wentz, IEEE Trans. Geosci. Remote Sens. 44, $506(2006)$

8. J.B. Masson, G. Gallot, Opt. Lett. 31, 265 (2006)

9. P. Fei, Z.X. Shen, X. Wen, N. Feng, IEEE Trans. Antennas Propag. 63, 4609 (2015)

10. B. Lin, J.L. Wu, X.Y. Da, W. Li, J.J. Ma, Appl. Phys. A 123, 43 (2017)
11. D.J. Liu, Z.Y. Xiao, X.L. Ma, Z.H. Wang, Opt. Commun. 354, 272 (2015)

12. M. Zalkovskij, R. Malureanu, C. Kremers, D.N. Chigrin, A. Novitsky, S. Zhukovsky, Laser Photonics Rev. 7, 810 (2013)

13. M. Euler, V. Fusco, R. Cahill, R. Dickie, IEEE Trans. Antennas Propag. 58, 2457 (2010)

14. Y. Yang, W. Wang, P. Moitra, I.I. Kravchenko, D.P. Briggs, J. Valentine, Nano Lett. 14, 1394 (2014)

15. L. Wu, Z. Yang, Y. Cheng, R. Gong, M. Zhao, Y. Zheng, Appl. Phys. A 116, 643 (2014)

16. M.A. Joyal, J.J. Laurin, IEEE Trans. Antennas Propag. 62, 3043 (2014)

17. I.L. Morrow, P. Thomas, Electron. Lett. 50, 64 (2014)

18. L. Li, Y. Li, Z. Wu, F. Huo, Y. Zhang, C. Zhao, Proc. IEEE 103, 1057 (2015)

19. L.B. Zhang, P. Zhou, H.Y. Chen, H.P. Lu, H.Y. Xie, L. Zhang, E. Li, J.L. Xie, L.J. Deng, Sci. Rep. 6, 33826 (2016)

20. S.M.A.M.H. Abadi, N. Behdad, IEEE Trans. Antennas Propag. 64, 525 (2016)

21. H. Cheng, S. Chen, P. Yu, J. Li, L. Deng, J. Tian, Opt. Lett. 38, 1567 (2013)

22. W. Geyi, IEEE Trans. Antennas Propag. 51, 2124 (2003)

23. P. Xu, W.X. Jiang, S.Y. Wang, T.J. Cui, IEEE Trans. Antennas Propag. 66, 4370 (2018)

24. R.E. Collin, S. Rothschild, IEEE Trans. Antennas Propag. 12, 23 (1964)

25. G.W. Ding, K. Chen, X.Y. Luo, J.M. Zhao, T. Jiang, Y.J. Feng, Phys. Rev. Appl. 11, 044043 (2019)

26. K. Zhang, Y.Y. Yuan, X.M. Ding, H.Y. Li, B. Ratni, Q. Wu, J, Liu, S.N. Burokur, J.B. Tan, Laser Photonics Rev. 10, $2000351(2020)$

27. Y.Y. Yuan, K. Zhang, B. Ratni, Q.H. Song, X.M. Ding, Q. Wu, S.N. Burokur, P. Genevet, Nat. Commun. 11, 4186 (2020)

28. Y.Y. Yuan, S. Sun, Y. Chen, K. Zhang, X.M. Ding, B. Ratni, Q. Wu, S.N. Burokur, C.W. Qiu, Adv. Sci. 7, 2001437 (2020)

Cite this article as: Peng Xu, Wei Xiang Jiang, Xiao Cai, Yue Gou, Tie Jun Cui, A high-efficiency and ultrathin transmissiontype circular polarization converter based on surface structure, EPJ Appl. Metamat. 8, 4 (2021) 